\title{
Clinical practice of transarterial chemoembolization for hepatocellular carcinoma: consensus statement from an international expert panel of International Society of Multidisciplinary Interventional Oncology (ISMIO)
}

\author{
Jian Lu ${ }^{1 \#}$, Ming Zhao ${ }^{2,3 \#, ~ Y a s u a k i ~ A r a i ~}{ }^{4 \#}$, Bin-Yan Zhong ${ }^{5 \#}$, Hai-Dong Zhu ${ }^{1 \#, ~ X i a o-L o n g ~ Q i ~}{ }^{6}$, \\ Thierry de Baere ${ }^{7}$, Uei Pua ${ }^{8}$, Hyun Ki Yoon ${ }^{9}$, David C. Madoff ${ }^{10}$, Gao-Jun Teng ${ }^{1}$; International Society of \\ Multidisciplinary Interventional Oncology (ISMIO)
}

${ }^{1}$ Center of Interventional Radiology \& Vascular Surgery, Department of Radiology, Zhongda Hospital, Medical School, Southeast University, Nanjing, China; ${ }^{2}$ State Key Laboratory of Oncology in Southern China, Guangzhou, China; ${ }^{3}$ Minimally Invasive \& Interventional Department, Cancer Center, Sun Yat-sen University, Guangzhou, China; ${ }^{4}$ Department of Diagnostic Radiology, National Cancer Center, Tokyo, Japan; ${ }^{5}$ Department of Interventional Radiology, The First Affiliated Hospital of Soochow University, Suzhou, China; ${ }^{6}$ CHESS Frontier Center, First Hospital of Lanzhou University, Lanzhou, China; ${ }^{7}$ Gustave Roussy-Cancer Campus, Villejuif, France; ${ }^{8}$ Department of Radiology, Tan Tock Seng Hospital, National University of Singapore, Singapore, Singapore; ${ }^{9}$ Department of Radiology and Research Institute of Radiology, University of Ulsan College of Medicine, Asan Medical Center, Seoul, Korea; ${ }^{10}$ Department of Radiology and Biomedical Imaging, Section of Interventional Radiology and Department of Internal Medicine, Section of Medical Oncology, Yale School of Medicine, New Haven, CT, USA

Contributions: (I) Conception and design: All authors; (II) Administrative support: None; (III) Provision of study materials or patients: None; (IV) Collection and assembly of data: None; (V) Data analysis and interpretation: None; (VI) Manuscript writing: All authors; (VII) Final approval of manuscript: All authors.

\#These authors contributed equally to this work as the joint first authors.

Correspondence to: Gao-Jun Teng, MD. Center of Interventional Radiology \& Vascular Surgery, Department of Radiology, Zhongda Hospital, Southeast University, 87 Dingjiaqiao Road, Nanjing 210009, China. Email: gjteng@seu.edu.cn; David C. Madoff, MD. Department of Radiology and Biomedical Imaging, Section of Interventional Radiology and Department of Internal Medicine, Section of Medical Oncology, Yale School of Medicine, New Haven, CT 06520, USA. Email: david.madoff@yale.edu.

Importance: Transarterial chemoembolization (TACE) has been associated with a wide range of practice variations for hepatocellular carcinoma (HCC) between the East and the West. This considerable ambiguity may lead to the heterogeneous quality in treatment and have a negative impact on the role of TACE in the overall multidisciplinary HCC treatment system.

Objective: It may be a good start to establish a guideline worldwide to have this consensus from experts who represent east and west, although it does not cover all aspects of TACE.

Evidence Review: An international expert panel on TACE is convened to cluster the expert's opinions and summary a standard consensus. This panel committee consist of leading physicians in TACE on HCC from USA, France, Japan, Singapore, Korea, China, and so on. The first-round face-to-face consensus meeting was held during in Nanjing, China in October 2019. The second-round conference for revision of the consensus was held during the Annual Meeting of Chinese College of Interventionalists in August 2020 by a hybrid format of a Webinar and roundtable meeting. After several on-line revisions, the final manuscript was approved by all members of the panel in June 2021.

Findings: The consensus statements were organized into the following categories: patients' selection, performing the procedure, TACE outcomes, repeat TACE, TACE failure/refractory, and TACE-based combination treatments.

Conclusions and Relevance for Reviews: More and more evidences have showed the better outcomes with strategy of combined TACE with other local therapies such as ablations. The most-recently 


\begin{abstract}
developing strategy of combined TACE with PD-1/PD-L1 plus tyrosine kinase inhibitor (TKI) agents has shined a light to the HCC patients, especially to those with high risk of tumor recurrence after treatment or TACE failure/refractory.
\end{abstract}

Keywords: Transarterial chemoembolization (TACE); hepatocellular carcinoma (HCC); consensus

Submitted Jun 24, 2021. Accepted for publication Aug 02, 2021.

doi: 10.21037/hbsn-21-260

View this article at: https://dx.doi.org/10.21037/hbsn-21-260

\section{Introduction}

Liver cancer is the sixth most diagnosed cancer and the third leading cause of cancer-related death worldwide in 2020 (1). Asia accounts for nearly $72.5 \%$ of the newly diagnosed cases and $72.4 \%$ of the deaths. In addition, a rising trend of incidence has been observed in most countries of Europe, the Americas, and Oceania over recent decades (2). Hepatocellular carcinoma (HCC) typically represents nearly $85 \%$ of all liver cancer cases. The prognosis of HCC remains dismal with the maximum 5 -year survival estimated at $18 \%$ and thus poses a heavy health burden globally (2). As a pillar technique of interventional radiology, transarterial chemoembolization (TACE) plays a fundamental component in the management of HCC.

TACE has been associated with a wide range of practice pattern variations for HCC between the East and the West, and there is even considerable variability among the physicians practicing within the same institution (3-6). This considerable ambiguity may lead to the heterogeneous quality in treatment and have a negative impact on the role of TACE in the overall multidisciplinary HCC treatment system.

Therefore, to optimize the TACE practice and improve the care of HCC patients, an international expert panel on TACE was convened to cluster the expert's opinions to provide a standard consensus. This panel committee consisted of leading physicians in TACE on HCC from the USA, France, Japan, Singapore, Korea, and China. The first-round face-to-face consensus meeting was held during in Nanjing, China in October 2019 and the panel decided to write a global expert consensus statement on TACE practice. The second-round conference for revision of the consensus was held during the Annual Meeting of the Chinese College of Interventionalists (CCI 2020) in August 2020 by a hybrid format of a webinar and roundtable meeting. After several subsequent on-line revisions, the final manuscript was approved by all members of the panel in
February 2021. The consensus statements were organized into the following categories: patients' selection, procedural technique, TACE outcomes, repeat TACE and TACE failure/refractory, and TACE-based combination treatments (Table 1). We present the following article in accordance with the RIGHT reporting checklist (available at https:// hbsn.amegroups.com/article/view/10.21037/hbsn-21-260/rc).

\section{Patient selection}

According to recommendations from the Barcelona Clinic Liver Cancer (BCLC) staging system, intermediate stage HCC is the main indication for TACE. However, a global survey entitled the "HCC BRIDGE Study" showed that TACE was the most frequently used first-line treatment across all BCLC stages (BCLC A-D) in North America, Europe, China, and South Korea; with $51 \%$ of HCC patients from China and $35 \%$ from other Asian regions receiving TACE as the initial treatment for HCC (7). Even after first resection or ablation with relapse or high-risk patients, TACE was still the most frequently used therapy (48-72\%) in those HCC population in Asian regions, which seemed higher than that in the West (31-43\%) (7).

TACE is often chosen as a bridge therapy prior to liver transplantation (LT) when the anticipated waiting time for receiving an organ is longer than 6 months, and has been associated with a decrease in the dropout rate for patients waiting for LT. Patients with BCLC-A stage HCC who are not candidates for surgical resection or ablation can also benefit from the treatment of TACE for its effective tumor response and clinical outcome $(8,9)$. TACE has also been combined with portal vein embolization prior to resection to increase the rate of future liver remnant hypertrophy and to obtain a high rate of complete tumor necrosis (10).

BCLC-B stage represents a wide group of patients with a variety of tumor burdens and liver functions. Indications of TACE for BCLC-B stage are mainly patient populations with massive or multinodular tumors but without 
Table 1 Summary of recommendations for TACE in HCC

\begin{tabular}{ll}
\hline Categories & Consensus statements \\
\hline No. 1 Patient selection & TACE is indicated for those patients who belong to BCLC-B, Eastern Cooperative Oncology Group \\
& $($ ECOG) performance status (PS) $\leq 2$, Child-Pugh A and B. For BCLC-A patients, TACE is recommended \\
& if surgical resection or ablation is impractical or refused by patient. For BCLC-C patients with liver \\
& dominant lesions, including those with macrovascular invasion, TACE is recommended as an option if \\
the Child-Pugh $<$ C and PS are tolerant
\end{tabular}

No. 2 Tumor targeting

Selective or super-selective vessel catheterization should be achieved as much as technically feasible to target the tumor, and three-dimension CTA images with Angio-CT system or cone-beam CT is recommended for TACE

No. 3 Chemotherapy regimen of CTACE

No. 4 Chemotherapeutic drugs for DEB-TACE

No. 5 TACE endpoint

No. 6 Conventional vs. DEBTACE

No. 7 Repeat TACE, TACE failure/refractory

No. 8 The combination of TACE and local therapy

No. 9 Combined TACE with systemic therapy
Doxorubicin is the fundamental chemotherapeutic agent for TACE, either in combination with cisplatin, mitomycin-C, or other drugs. Dosage of ethiodized oil administered should be tumor-based volume

At present, various cytotoxic agents alone or in combination with other drugs are used for DEBTACE while doxorubicin is the most widely used chemotherapeutic agent. However, there is no clinical evidence to suggest any superiority of doxorubicin-DEB-TACE over epirubicin-DEB-TACE over for HCC

The endpoint of TACE is defined as a "tree in winter" appearance in case of non-selective TACE. Filling in the peritumoral portal veins around the tumor must be attempted in CTACE while reflux of embolic materials must be avoided in DEB-TACE

At present, none of the RCTs have showed significant OS differences between DEB-TACE and cTACE. DEB-TACE has less systemic toxicity, but more bile duct injury. Nevertheless, there are some specific clinical settings to choose DEB-TACE or CTACE in the real-world practice, including huge tumors or bilobar tumors, hypovascular tumors, or HCC with PVTT

TACE should be performed according to the "on demand" approach and not on schedule. The concepts of "TACE failure/refractoriness" have been introduced although high-level evidence is still lacking

The combination of TACE with thermal ablative techniques may result in better outcomes than ablation or TACE monotherapy in selected populations of early- or intermediate-HCC. For patients with macrovascular invasion, TACE plus radiotherapy may be an alternative choice to systemic therapies.

TACE plus systemic therapy with an appropriate protocol may improve the therapeutic outcomes in the treatment of unresectable $\mathrm{HCC}$

BCLC, Barcelona Clinic Liver Cancer; CTA, computed tomography angiography; DEB, drug-eluting bead; HCC, hepatocellular carcinoma; OS, overall survival; PVTT, portal vein tumor thrombosis; RCT, randomized controlled trial; TACE, transarterial chemoembolization; cTACE, conventional TACE.

macrovascular invasion or extrahepatic metastasis $(5,6)$. TACE can function as a downstaging treatment in patients with BCLC-B stage to meet the threshold of surgical resection, and/or LT (11).

Advanced HCC, with vascular invasion or extrahepatic metastasis, is typically considered a contraindication for TACE according to the BCLC staging system (4). However, a meta-analysis demonstrated that only $1 \%$ of patients developed liver failure and 18\% acquired post-embolization complications after TACE, and the median overall survival (OS) was 11 months for patients with segmental portal vein tumor thrombosis (PVTT) and 5 months for those with main PVTT (12). For patients with limited extrahepatic metastasis with liver-dominant disease, who are most likely to die from intrahepatic disease, TACE may still play a role in controlling the intrahepatic tumors (13). For selected Child-Pugh C patients within Milan criteria, TACE is also performed in Asia (14). With the accumulation of clinical evidence, more and more experts are advocating to expand the indications of TACE for selected patients with advanced stage in the BCLC treatment algorithm.

\section{Consensus No. 1}

TACE is indicated for those patients who belong to BCLC-B, Eastern Cooperative Oncology Group (ECOG) performance status (PS) $\leq 2$, Child-Pugh A and B. For BCLC-A patients, TACE is recommended if surgical 
resection or ablation is impractical or refused by patient. For BCLC-C patients with liver dominant lesions, including those with macrovascular invasion, TACE is recommended as an option if the Child-Pugh $<\mathrm{C}$ and PS are tolerant.

\section{Procedural technique}

\section{Tumor targeting}

Adequate visualization of all tumor-feeding feeding arteries should be obtained during the procedure, including vessels' origin, variant anatomy, and ectopic or collateral blood supply. Computed tomography (CT) during arteriography and advanced imaging technique with conebeam CT/3D rotational angiography, Angio-CT system, or other software guidance are advocated to best delineate the vascular anatomy and/or tumor supply/evaluation for additional tumor supply/assessment completeness of embolization (15). Regarding catheter position for embolization, catheterization within selective or superselective tumor feeding arteries should be always achieved as much as possible (16).

\section{Consensus No. 2}

Selective or super-selective vessel catheterization should be achieved as much as technically feasible to target the tumor, and three-dimension computed tomography angiography (CTA) images with Angio-CT system or cone-beam CT is recommended for TACE.

\section{Chemotherapy regimen of conventional TACE (cTACE)}

Chemotherapy regimen selection varies from center to center, whether in the East or in the West. Doxorubicin is the most common single chemotherapeutic drug, while the most common combination regimens is doxorubicin and cisplatin (17). In addition, mitomycin C, hydroxycamptothecin, fluorouracil, arsenic trioxide, and raltitrexed serve as the alternative agents. However, the scenarios and indications for such alternatives have yet to be determined. The dosage of chemotherapeutic drug used could be body surface area-based, liver function-based, weight-based, or even empiric. Currently used dose ranges as follows: doxorubicin, 10-100 mg; epirubicin, 5-120 mg; cisplatin, 10-100 mg; miriplatin, 20-140 mg; mitomycin, 2-30 mg, and idarubicin, 10-20 mg (17). The ethiodized oil and chemotherapeutic drugs should be mixed into an emulsion and configured as a "water-in-oil" emulsifier to improve its stability. The volume ratio of ethiodized oil to drug aqueous solution is usually 2:1. Doxorubicinethiodized oil emulsions prepared with nonionic contrast medium would be safer and more stable compared to be prepared with ionic contrast medium (18). The volume of ethiodized oil injected is generally determined by the size and vascularity of the tumor, with common usage of $5-15 \mathrm{~mL}$, with increased risk of complication above $20 \mathrm{~mL}(5)$.

Particulate embolic agents (e.g., standardized gelatin sponge particles, microspheres, polyvinyl alcohol particles) should be used following embolization with ethiodized oil chemoembolic emulsion. The size of embolic materials should be based on the tumor size, blood supply, and therapeutic aim. Gelatin sponge particles measuring 500 mm and blank microspheres measuring 100-300 mm have been widely used (19). All tumor-feeding arteries should be embolized to achieve devascularization of the tumor.

\section{Consensus No. 3}

Doxorubicin is the fundamental chemotherapeutic agent for TACE, either in combination with cisplatin, mitomycin-C, or other drugs. Dosage of ethiodized oil administered should be tumor-based volume.

\section{Chemotherapeutic drugs for drug-eluting bead (DEB)- TACE}

DEB-TACE releases chemotherapeutic drugs in a controlled and sustained manner. By now, the most widely used cytotoxic agent for DEB-TACE is doxorubicin; however, other anthracycline drugs such as epirubicin or idarubicin that can be loaded into drug-eluting microspheres (20). The value of doxorubicin-DEB-TACE in the setting of enhancing the local drug concentration was investigated in a randomized trial testing DEB-TACE $v s$. bland embolization. Better outcomes, including local response, local recurrences, and time to progression was achieved in patients treated with DEB-TACE than bland embolization alone (21). On the other hand, another randomized controlled trial (RCT) demonstrated that there were no significant differences in the tumor response and survival between the DEB-TACE and bland embolization groups (22).

Epirubicin, an epimer of doxorubicin which was commonly considered having less myelosuppression and cardiac toxicities than doxorubicin, has also been used in clinical trials of HCC patients treated with DEB-TACE and 
compared to bland-transarterial embolization (TAE) (23). Seventy-seven percent of lesions treated with epirubicinDEB-TACE achieved complete necrosis, whereas only $27.2 \%$ of lesions in the bland-TACE group showed such responses $(\mathrm{P}=0.043)$ (23). At present, no study exists that compared the clinical benefits between epirubicin-DEBTACE with doxorubicin-DEB-TACE in patients with HCC. To that end, further studies involving epirubicinDEB-TACE for HCC patients should be cautiously designed because there were limiting data from the preclinical study that testing the pharmacokinetic and safety of epirubicin-beads in hepatic arterial embolization.

\section{Consensus No. 4}

At present, various cytotoxic agents alone or in combination with other drugs are used for DEB-TACE while doxorubicin is the most widely used chemotherapeutic agent. However, there is no clinical evidence to suggest any superiority of doxorubicin-DEB-TACE over epirubicinDEB-TACE over for HCC.

\section{TACE endpoint}

The endpoint of TACE is defined at angiography as the presence of flow stasis of the tumor feeding arteries under fluoroscopic monitoring. Complete stasis is visualized as a static contrast column for at least 5 heartbeats. For cTACE, the optimal complete embolization is featured by the appearance of the lipiodol filling of in periphery portal veins around the tumor (24). For DEB-TACE, injection must be stopped once stasis is observed in the tumor feeding arteries to avoid reflux of embolic materials (25).

\section{Consensus No. 5}

The endpoint of TACE is defined as a "tree in winter" appearance in case of non-selective TACE. Filling in the peritumoral portal veins around the tumor must be attempted in cTACE while reflux of embolic materials must be avoided in DEB-TACE.

\section{TACE outcomes}

In 2002, the Asian RCT reported that patients with unresectable HCC who in the cTACE cohort had a $57 \%$, $31 \%$, and $26 \%$ survival rate at 1,2 and 3 years compared to a $32 \%, 11 \%$, and $3 \%$, respectively $(\mathrm{P}=0.002)$ survival rate in patients who received symptomatic therapy (26). Similarly, a European cohort demonstrated that the survival rate at 1 and 2 years with cTACE was $82 \%$ and $63 \%$ which was significantly higher than the symptomatic therapy which had a $63 \%$ and $27 \%$ at 1 and 2 years $(\mathrm{P}=0.025)$ (27). An Asian prospective study enrolled 99 patients with unresectable HCC from Japan and Korea. The 2-year survival rate achieved $75.0 \%$ (95\% CI, $65.2-82.8 \%)$. The median OS was 3.1 years. And the response rate was $73 \%$ using modified Response Evaluation Criteria in Solid Tumors (mRECIST) (28).

In a systematic review, including a total of 10,108 patients treated with cTACE, the OS was $70.3 \%$ at 1 year, $51.8 \%$ at 2 years, $40.4 \%$ at 3 years, and $32.4 \%$ at 5 years. Median OS was 19.4 months (17). These data further confirmed the efficacy of cTACE from historical RCTs and meta-analyses. Unfortunately, in such a high heterogeneity population, the outcomes of subgroup survival analysis based on BCLC stage, Child-Pugh class, PS, etc., were not stratified due to insufficient data.

\section{cTACE vs. DEB-TACE}

Since DEB-TACE first became commercially available in 2006, multiple clinical trials have been conducted to confirm its theoretical superiority in efficacy over cTACE. In 2010, the randomized phase II PRECISION V study first reported the results comparing DEB-TACE with cTACE for patients with unresectable HCC (29). The disease control rates in the DEB-TACE group failed to reach a statistical significance when compared to those in the cTACE group $(63.4 \%$ vs. $51.9 \%, \mathrm{P}=0.11)$. Notably, in a post-hoc analysis, $67 \%$ of patients with more advanced disease (Child-Pugh B, ECOG 1, bilobar or recurrent disease) had a significant increase in objective response and disease control $(\mathrm{P}=0.038$ and $\mathrm{P}=0.026$, respectively) in the DEB-TACE group compared with the cTACE group. Furthermore, DC Beads reduced the rate of serious liver toxicity $(\mathrm{P}<0.001)$ and doxorubicin-related side effects $(\mathrm{P}=0.0001)$ (29). On the other hand, increase in liver parenchyma damage has been reported for DEB-TACE $v s$. cTACE, namely in patient with the lower degree of cirrhosis (30).

A subsequent multicenter RCT reported the survival and safety comparison between the doxorubicin-loaded DEBTACE and cTACE in patients with unresectable HCC. The 1- and 2-year survival rates were $86.2 \%$ and $56.8 \%$ after DEB-TACE and $83.5 \%$ and $55.4 \%$ after cTACE $(\mathrm{P}=0.949)$ and the only distinct advantage of DEB-TACE was its lower rate of post-procedural abdominal pain $(71.6 \%$ 
in the cTACE group vs. $24.7 \%$ in the DEB-TACE group, $\mathrm{P}=0.001)$ (31).

Interestingly, an updated meta-analysis including 1,449 patients from four RCTs and eight retrospective studies demonstrated that non-superiority was observed in DEBTACE when compared with cTACE in terms of tumor response and survival (32). Recently, a multi-center RCT demonstrated that cTACE appeared to have greater efficacy for local tumor control as compared to DEB-TACE (complete response rate at 3 months: $75.2 \%$ in cTACE group vs. $27.6 \%$ in DEB-TACE group, $\mathrm{P}<0.0001$ ) (33). It should be indicated that $74.3 \%$ and $73.5 \%$, respectively patients in cTACE and DEB-TACE group in this study had small tumor burden of $\leq 30 \mathrm{~mm}$ (33).

\section{Consensus No. 6}

At present, none of the RCTs have shown significant OS differences between DEB-TACE and cTACE. DEBTACE has less systemic toxicity, but more bile duct injury. Nevertheless, there are some specific clinical settings to choose DEB-TACE or cTACE in the real-world practice, including huge tumors or bilobar tumors, hypovascular tumors, or HCC with PVTT.

\section{Repeat TACE, TACE failure/refractory}

The purpose of TACE for patients with HCC is to locally control or shrink the tumor. Several previous studies demonstrated that the repetition of cTACE maximizes tumor response as well as prolongs OS (34). Repeated TACE could be associated with liver damage and increased side effects. Therefore, a delicate balance between the necessity of repeat TACE and treatment benefit should be considered. Two major modes of therapy, classified as "scheduled" or "on-demand" modes, are now applied in clinical practice, with the latter one more widely accepted. For the "scheduled" approach, TACE is performed and repeated at fixed intervals even if intrahepatic tumors respond favorably. However, this approach may result in impaired liver function as a result of unnecessary repeated procedures. For the "on-demand" approach, repeat TACE is performed only upon the demonstration of a viable tumor (non-complete response) or local and/or distant intrahepatic recurrences for patients with preserved liver function. For the "on-demand" approach, TACE should also be repeated if partial response/stable disease has been achieved from the previous TACE, which is paradoxical to the palliative nature of TACE. In addition, based on a previous study, at least two
TACE sessions to a particular tumor, should be performed before assessing general tumor response, since $47 \%$ patients who did not respond to the first TACE ultimately achieved complete response or partial response, and the OS did not differ significantly between patients who achieved response in the first and second session (35). Notably, a qualitative definition rather than a quantitative definition is applied in "on-demand" mode when assessing tumor response. Several predictive models such as ART (Assessment for Retreatment with TACE) score and ABCR ( $\alpha$-fetoprotein, BCLC, ChildPugh, and response) score have been established to assess and determine the survival benefit of repeat TACE during the past years (36). Nevertheless, none of them has been widely applied in clinical practice.

To ensure that patients receive continuous benefit from repeated TACE and in an attempt to avoid ineffective repeat treatments, several organizations, including the Japan Society of Hepatology (JSH), the European Association for the Study of the Liver (EASL), and the International Association for the Study of the Liver, introduced the concept of "TACE failure/refractoriness" (37). Among them, the updated version by the JSH-Liver Cancer Study Group of Japan (LCSGJ) in 2014 was most widely introduced in clinical trials, of which TACE failure/refractoriness was defined by intra-hepatic progression or vascular/extra-hepatic invasion after one or two TACE sessions (38). Mainly based on the results of the OPTIMIS trial, the concept suggests switching to systemic agent monotherapy if "TACE failure/refractoriness" occurs (39). By contrast, several studies demonstrated that repeat TACE especially TACE combination therapy can also achieve survival benefit $(34,40)$. In addition, the rationality of "TACE failure/refractoriness" concept needs further discussion. Further studies with high-level evidence are warranted to demonstrate subsequent treatment after failure of TACE.

\section{Consensus No. 7}

TACE should be performed according to the "on demand" approach and not on schedule. The concepts of "TACE failure/refractoriness" have been introduced although highlevel evidence is still lacking.

\section{The combination of TACE and local therapy}

\section{TACE combined with thermal ablation}

For patients with early-stage HCC, further TACE use might improve the survival outcome of thermal ablation 
such as radiofrequency ablation (RFA) and microwave ablation (MWA). A RCT demonstrated that RFA plus TACE could decrease the rate of recurrence compared to RFA alone for early-stage HCC with characteristics of a solitary nodule no more than $7 \mathrm{~cm}$ in diameter or multiple (three or fewer) lesions with each diameter no more than $3 \mathrm{~cm}(35.11 \%$ vs. $54.74 \%)$. Patients in the TACE-RFA group had better OS and recurrence-free survival (RFS) than those in the RFA group $(\mathrm{P}=0.002 ; \mathrm{P}=0.009)$ (41). Chu et al. reported a propensity-score analysis comparing the OS of patients with single medium-sized $(3.1-5.0 \mathrm{~cm})$ HCCs who underwent first-line treatment with TACE, RFA, or a combination of the two therapies (42). The 10 -year OS rates were significantly different among the three groups (the poor cohort, $40.1 \%$ with combined therapy, $25.5 \%$ with TACE alone, and $19.5 \%$ with RFA alone; the matched cohort, $41.8 \%, 28.4 \%$, and $11.9 \%$, respectively; $\mathrm{P}=0.022)$. In the treatment of recurrent early-stage HCC, TACE-RFA combined therapy also showed better efficacy than RFA monotherapy. The 1-, 3 -, and 5-year RFS rates in a RCT were $80 \%, 45 \%$, and $40 \%$ for the combined group and 64\%, $18 \%$, and $18 \%$ for the control group $(\mathrm{P}=0.005)$ (43). Recently, a metaanalysis, including eight retrospective studies and one RCT, compared the efficacy and survival outcome between TACE plus RFA and surgical resection in patients with early-stage HCC. The combined therapy showed similar OS, diseasefree survival, intrahepatic distant recurrence rate and distant metastasis rate to the resection therapy, but showed a higher local tumor progression rate [objective response rate (ORR) 2.48, 95\% CI, 1.05-5.86, $\mathrm{P}=0.04]$ (44). Patients treated with MWA of HCC after downstaging with TACE obtain similar OS and RFS to those initially met Milan criteria (45).

\section{TACE combined with radiotherapy (RT)}

In the past decade, an increasing number of studies have indicated the therapeutic benefit of TACE combined with RT for HCC. A recent RCT showed that first-line treatment with TACE plus RT provides longer OS (55.0 vs. 43.0 weeks; $\mathrm{P}=0.04)$ and time to disease progression $(31.0$ vs. 11.7 weeks; $\mathrm{P}<0.001)$ than sorafenib for HCC patients with macroscopic vascular invasion (46).

Recently, a propensity score-matching study revealed that TACE plus brachytherapy with iodine-125 implantation significantly improved local control and survival in patients with 3-5 cm HCC (47). A meta-analysis demonstrated that HCC patients receiving combined therapy of TACE plus iodine-125 implantation obtained higher 1- and 2-year OS $(\mathrm{P}<0.001)$ than TACE monotherapy (48).

\section{Consensus No. 8}

The combination of TACE with thermal ablative techniques may result in better outcomes than ablation or TACE monotherapy in selected populations of early or intermediate HCC. For patients with macrovascular invasion, TACE plus RT may be an alternative choice to systemic therapies.

\section{Combined TACE with systemic therapy}

As a potent multikinase inhibitor that targets vascular endothelial growth factor (VEGF) receptors, sorafenib has been expected to induce a synergistic action when combining with TACE for HCC.

Unfortunately, in the past decade, series of multi-center RCTs including the post-TACE trial (49), the SPACE trial (50), the TACE-2 trial (51), and the STAH trial (52) failed to demonstrate the expected synergistic results regarding TACE combined with sorafenib $v s$. TACE alone for unresectable HCC. TACE combined with other molecular targeted agent such as with brivanib (the BRISKTA trial) and with orantinib (the ORIENTAL trial) also showed negative treatment benefit $(53,54)$.

More recently, the TACTICS trial demonstrated positive treatment efficacy for TACE when combined with sorafenib compared to TACE alone for unresectable HCC. The primary endpoint of progression-free survival was significantly longer in the combination group. Different from the previously mentioned trials above, sorafenib was earlier administered pre-treatment with TACE. Time to untreated progression (TTUP) was proposed as a new primary endpoint in TACE combination trials with systemic therapy (55). The ideal candidates or prognostic biomarkers of TACE combined with molecular targeted agents for HCC is still uncertain.

Following the recent successes of targeting immune checkpoints in various malignancies, the immunotherapy era for HCC treatment has begun. The CheckMate-040 trial with Nivolumab, KEYNOTE-224 trial with Pembrolizumab, and IMBRAVE 150 trial with atezolizumab plus bevacizumab in advanced HCC has shown promising outcomes (56-58). Several phase I or II trial have been underway to evaluate the safety and efficacy of TACE plus nivolumab (NCT03143270, NCT03572582, 
NCT04268888). Similarly, the safety and efficacy of TACE plus pembrolizumab is also being studied in a phase I/II trial (NCT03397654). There are some phase III RCTs in TACE-based combination investigation in patients with HCC, such as LEAP-012 trial (NCT04246177) using lenvatinib plus pembrolizumab vs. placebo in combination with TACE, CheckMate 74W trial (NCT04340193) using nivolumab and ipilimumab plus TACE, and EMERALD study (NCT03778957) using durvalumab and bevacizumab plus TACE. These ongoing studies deserve further evaluation and may open a new chapter of TACE combined with systemic therapies.

\section{Consensus No. 9}

TACE plus systemic therapy with an appropriate protocol may improve the therapeutic outcomes in the treatment of unresectable HCC.

\section{Perspective}

Although the use of TACE has over 40 years of history, none of an international consensus or guideline document on TACE technique has been published so far. Obviously, the heterogeneity of TACE technique exists extensively among the different regions, medical sites, and even colleagues within the same interventional radiologist team. Therefore, it may be a good start to establish a guideline worldwide to have this consensus from experts who represent East and West, although it does not cover all aspects of TACE. The present international consensus statement compromises the opinions between East and West experts who are from Northern America, Europe, and Asia-Pacific areas.

More and more evidences have shown the better outcomes with a strategy of combined TACE with other local therapies such as ablations. The most-recently developing strategy of combined TACE with PD-1/PDL1 plus tyrosine kinase inhibitor (TKI) agents has shined a light to the HCC patients, especially to those with high risk of tumor recurrence after treatment or TACE failure/ refractory.

\section{Acknowledgments}

Funding: The study was supported by Jiangsu Provincial Special Program of Medical Science (BE2019750), National Natural Science Foundation of China (81827805), and
National Key Research and Development Program (2018YFA0704100, 2018YFA0704104). The funding sources had no role in the writing of the report, or decision to submit the paper for publication.

\section{Footnote}

Reporting Checklist: The authors have completed the RIGHT reporting checklist. Available at https://hbsn. amegroups.com/article/view/10.21037/hbsn-21-260/rc

Conflicts of Interest: All authors have completed the ICMJE uniform disclosure form (available at https://hbsn. amegroups.com/article/view/10.21037/hbsn-21-260/coif). The authors report grants from Jiangsu Provincial Special Program of Medical Science (BE2019750), National Natural Science Foundation of China (81827805), and National Key Research and Development Program (2018YFA0704100, 2018YFA0704104).

Ethical Statement: The authors are accountable for all aspects of the work in ensuring that questions related to the accuracy or integrity of any part of the work are appropriately investigated and resolved.

Open Access Statement: This is an Open Access article distributed in accordance with the Creative Commons Attribution-NonCommercial-NoDerivs 4.0 International License (CC BY-NC-ND 4.0), which permits the noncommercial replication and distribution of the article with the strict proviso that no changes or edits are made and the original work is properly cited (including links to both the formal publication through the relevant DOI and the license). See: https://creativecommons.org/licenses/by-nc-nd/4.0/.

\section{References}

1. Sung H, Ferlay J, Siegel RL, et al. Global Cancer Statistics 2020: GLOBOCAN Estimates of Incidence and Mortality Worldwide for 36 Cancers in 185 Countries. CA Cancer J Clin 2021;71:209-49.

2. Arnold M, Abnet CC, Neale RE, et al. Global Burden of 5 Major Types of Gastrointestinal Cancer. Gastroenterology 2020;159:335-349.e15.

3. Marrero JA, Kulik LM, Sirlin CB, et al. Diagnosis, Staging, and Management of Hepatocellular Carcinoma: 2018 Practice Guidance by the American Association for the Study of Liver Diseases. Hepatology 2018;68:723-50. 
4. European Association for the Study of the Liver. Electronic address: easloffice@easloffice.eu; European Association for the Study of the Liver. EASL Clinical Practice Guidelines: Management of hepatocellular carcinoma. J Hepatol 2018;69:182-236.

5. Zhou J, Sun HC, Wang Z, et al. Guidelines for Diagnosis and Treatment of Primary Liver Cancer in China (2017 Edition). Liver Cancer 2018;7:235-60.

6. Kokudo N, Hasegawa K, Akahane M, et al. Evidencebased Clinical Practice Guidelines for Hepatocellular Carcinoma: The Japan Society of Hepatology 2013 update (3rd JSH-HCC Guidelines). Hepatol Res 2015. doi: 10.1111/hepr.12464.

7. Park JW, Chen M, Colombo M, et al. Global patterns of hepatocellular carcinoma management from diagnosis to death: the BRIDGE Study. Liver Int 2015;35:2155-66.

8. Bargellini I, Sacco R, Bozzi E, et al. Transarterial chemoembolization in very early and early-stage hepatocellular carcinoma patients excluded from curative treatment: a prospective cohort study. Eur J Radiol 2012;81:1173-8.

9. Kang YJ, Lee BC, Kim JK, et al. Conventional Versus Small Doxorubicin-eluting Bead Transcatheter Arterial Chemoembolization for Treating Barcelona Clinic Liver Cancer Stage 0/A Hepatocellular Carcinoma. Cardiovasc Intervent Radiol 2020;43:55-64.

10. Ronot M, Cauchy F, Gregoli B, et al. Sequential transarterial chemoembolization and portal vein embolization before resection is a valid oncological strategy for unilobar hepatocellular carcinoma regardless of the tumor burden. HPB (Oxford) 2016;18:684-90.

11. Shi F, Wu M, Lian SS, et al. Radiofrequency Ablation Following Downstaging of Hepatocellular Carcinoma by Using Transarterial Chemoembolization: Long-term Outcomes. Radiology 2019;293:707-15.

12. Silva JP, Berger NG, Tsai S, et al. Transarterial chemoembolization in hepatocellular carcinoma with portal vein tumor thrombosis: a systematic review and meta-analysis. HPB (Oxford) 2017;19:659-66.

13. Cheng AL, Amarapurkar D, Chao Y, et al. Re-evaluating transarterial chemoembolization for the treatment of hepatocellular carcinoma: Consensus recommendations and review by an International Expert Panel. Liver Int 2014;34:174-83.

14. Choi TW, Kim HC, Lee JH, et al. The Safety and Clinical Outcomes of Chemoembolization in Child-Pugh Class C Patients with Hepatocellular Carcinomas. Korean J Radiol 2015;16:1283-93.
15. Miyayama S, Yamashiro M, Sugimori N, et al. Outcomes of Patients with Hepatocellular Carcinoma Treated with Conventional Transarterial Chemoembolization Using Guidance Software. J Vasc Interv Radiol 2019;30:10-8.

16. Miyayama S, Matsui O. Superselective Conventional Transarterial Chemoembolization for Hepatocellular Carcinoma: Rationale, Technique, and Outcome. J Vasc Interv Radiol 2016;27:1269-78.

17. Lencioni R, de Baere T, Soulen MC, et al. Lipiodol transarterial chemoembolization for hepatocellular carcinoma: A systematic review of efficacy and safety data. Hepatology 2016;64:106-16.

18. Tzeng WS, Wu RH, Chang SC, et al. Ionic versus nonionic contrast media solvents used with an epirubicinbased agent for transarterial chemoembolization of hepatocellular carcinoma. J Vasc Interv Radiol 2008;19:342-50.

19. Takayasu K. Transarterial chemoembolization for hepatocellular carcinoma over three decades: current progress and perspective. Jpn J Clin Oncol 2012;42:247-55.

20. Lewis AL, Gonzalez MV, Lloyd AW, et al. DC bead: in vitro characterization of a drug-delivery device for transarterial chemoembolization. J Vasc Interv Radiol 2006;17:335-42.

21. Malagari K, Pomoni M, Kelekis A, et al. Prospective randomized comparison of chemoembolization with doxorubicin-eluting beads and bland embolization with BeadBlock for hepatocellular carcinoma. Cardiovasc Intervent Radiol 2010;33:541-51.

22. Brown KT, Do RK, Gonen M, et al. Randomized Trial of Hepatic Artery Embolization for Hepatocellular Carcinoma Using Doxorubicin-Eluting Microspheres Compared With Embolization With Microspheres Alone. J Clin Oncol 2016;34:2046-53.

23. Nicolini A, Martinetti L, Crespi S, et al. Transarterial chemoembolization with epirubicin-eluting beads versus transarterial embolization before liver transplantation for hepatocellular carcinoma. J Vasc Interv Radiol 2010;21:327-32.

24. Miyayama S, Matsui O, Yamashiro M, et al. Ultraselective transcatheter arterial chemoembolization with a 2-f tip microcatheter for small hepatocellular carcinomas: relationship between local tumor recurrence and visualization of the portal vein with iodized oil. J Vasc Interv Radiol 2007;18:365-76.

25. Lencioni R, de Baere T, Burrel M, et al. Transcatheter treatment of hepatocellular carcinoma with Doxorubicin- 
loaded DC Bead (DEBDOX): technical recommendations. Cardiovasc Intervent Radiol 2012;35:980-5.

26. Lo CM, Ngan H, Tso WK, et al. Randomized controlled trial of transarterial lipiodol chemoembolization for unresectable hepatocellular carcinoma. Hepatology 2002;35:1164-71.

27. Llovet JM, Real MI, Montaña X, et al. Arterial embolisation or chemoembolisation versus symptomatic treatment in patients with unresectable hepatocellular carcinoma: a randomised controlled trial. Lancet 2002;359:1734-9.

28. Ikeda M, Arai Y, Park SJ, et al. Prospective study of transcatheter arterial chemoembolization for unresectable hepatocellular carcinoma: an Asian cooperative study between Japan and Korea. J Vasc Interv Radiol 2013;24:490-500.

29. Lammer J, Malagari K, Vogl T, et al. Prospective randomized study of doxorubicin-eluting-bead embolization in the treatment of hepatocellular carcinoma: results of the PRECISION V study. Cardiovasc Intervent Radiol 2010;33:41-52.

30. Monier A, Guiu B, Duran R, et al. Liver and biliary damages following transarterial chemoembolization of hepatocellular carcinoma: comparison between drug-eluting beads and lipiodol emulsion. Eur Radiol 2017;27:1431-9.

31. Golfieri R, Giampalma E, Renzulli M, et al. Randomised controlled trial of doxorubicin-eluting beads vs conventional chemoembolisation for hepatocellular carcinoma. Br J Cancer 2014;111:255-64.

32. Facciorusso A, Di Maso M, Muscatiello N. Drug-eluting beads versus conventional chemoembolization for the treatment of unresectable hepatocellular carcinoma: A meta-analysis. Dig Liver Dis 2016;48:571-7.

33. Ikeda $M$, Inaba $Y$, Tanaka $T$, et al. A prospective randomized controlled trial of selective transarterial chemoembolization using drug-eluting beads loaded with epirubicin versus selective conventional transarterial chemoembolization using epirubicin-lipiodol for hepatocellular carcinoma: The JIVROSG-1302 PRESIDENT study. J Clin Oncol 2020;38:abstr 4518.

34. Terzi E, Golfieri R, Piscaglia F, et al. Response rate and clinical outcome of HCC after first and repeated cTACE performed "on demand". J Hepatol 2012;57:1258-67.

35. Georgiades C, Geschwind JF, Harrison N, et al. Lack of response after initial chemoembolization for hepatocellular carcinoma: does it predict failure of subsequent treatment? Radiology 2012;265:115-23.
36. Sieghart W, Hucke F, Pinter M, et al. The ART of decision making: retreatment with transarterial chemoembolization in patients with hepatocellular carcinoma. Hepatology 2013;57:2261-73.

37. Park JW, Amarapurkar D, Chao Y, et al. Consensus recommendations and review by an International Expert Panel on Interventions in Hepatocellular Carcinoma (EPOIHCC). Liver Int 2013;33:327-37.

38. Kudo M, Matsui O, Izumi N, et al. Transarterial chemoembolization failure/refractoriness: JSH-LCSGJ criteria 2014 update. Oncology 2014;87 Suppl 1:22-31.

39. Peck-Radosavljevic M, Kudo M, Raoul JL, et al. Outcomes of patients (pts) with hepatocellular carcinoma (HCC) treated with transarterial chemoembolization (TACE): Global OPTIMIS final analysis. J Clin Oncol 2018;36:abstr 4018.

40. Zheng L, Fang S, Wu F, et al. Efficacy and Safety of TACE Combined With Sorafenib Plus Immune Checkpoint Inhibitors for the Treatment of Intermediate and Advanced TACE-Refractory Hepatocellular Carcinoma: A Retrospective Study. Front Mol Biosci 2021;7:609322.

41. Peng ZW, Zhang YJ, Chen MS, et al. Radiofrequency ablation with or without transcatheter arterial chemoembolization in the treatment of hepatocellular carcinoma: a prospective randomized trial. J Clin Oncol 2013;31:426-32.

42. Chu HH, Kim JH, Yoon HK, et al. Chemoembolization Combined with Radiofrequency Ablation for MediumSized Hepatocellular Carcinoma: A Propensity-Score Analysis. J Vasc Interv Radiol 2019;30:1533-43.

43. Peng ZW, Zhang YJ, Liang HH, et al. Recurrent hepatocellular carcinoma treated with sequential transcatheter arterial chemoembolization and RF ablation versus RF ablation alone: a prospective randomized trial. Radiology 2012;262:689-700.

44. Gui CH, Baey S, D'cruz RT, et al. Trans-arterial chemoembolization + radiofrequency ablation versus surgical resection in hepatocellular carcinoma - A metaanalysis. Eur J Surg Oncol 2020;46:763-71.

45. Shi F, Lian S, Mai Q, et al. Microwave ablation after downstaging of hepatocellular carcinoma: outcome was similar to tumor within Milan criteria. Eur Radiol 2020;30:2454-62.

46. Yoon SM, Ryoo BY, Lee SJ, et al. Efficacy and Safety of Transarterial Chemoembolization Plus External Beam Radiotherapy vs Sorafenib in Hepatocellular Carcinoma With Macroscopic Vascular Invasion: A Randomized Clinical Trial. JAMA Oncol 2018;4:661-9. 
47. Li M, He J, Pan M, et al. Iodine-125 implantation plus transarterial chemoembolization for the treatment of hepatocellular carcinoma of $3-5 \mathrm{~cm}$ : A propensity score matching study. Dig Liver Dis 2016;48:1082-7.

48. Zhu ZX, Wang XX, Yuan KF, et al. Transarterial chemoembolization plus iodine-125 implantation for hepatocellular carcinoma: a systematic review and metaanalysis. HPB (Oxford) 2018;20:795-802 .

49. Kudo M, Imanaka K, Chida N, et al. Phase III study of sorafenib after transarterial chemoembolisation in Japanese and Korean patients with unresectable hepatocellular carcinoma. Eur J Cancer 2011;47:2117-27.

50. Lencioni R, Llovet JM, Han G, et al. Sorafenib or placebo plus TACE with doxorubicin-eluting beads for intermediate stage HCC: The SPACE trial. J Hepatol 2016;64:1090-8.

51. Meyer T, Fox R, Ma YT, et al. Sorafenib in combination with transarterial chemoembolisation in patients with unresectable hepatocellular carcinoma (TACE 2): a randomised placebo-controlled, double-blind, phase 3 trial. Lancet Gastroenterol Hepatol 2017;2:565-75.

52. Park JW, Kim YJ, Kim DY, et al. Sorafenib with or without concurrent transarterial chemoembolization in patients with advanced hepatocellular carcinoma: The phase III STAH trial. J Hepatol 2019;70:684-91.

53. Kudo M, Han G, Finn RS, et al. Brivanib as adjuvant therapy to transarterial chemoembolization in patients with hepatocellular carcinoma: A randomized phase III

Cite this article as: Lu J, Zhao M, Arai Y, Zhong BY, Zhu HD, Qi XL, de Baere T, Pua U, Yoon HK, Madoff DC, Teng GJ; International Society of Multidisciplinary Interventional Oncology (ISMIO). Clinical practice of transarterial chemoembolization for hepatocellular carcinoma: consensus statement from an international expert panel of International Society of Multidisciplinary Interventional Oncology (ISMIO). HepatoBiliary Surg Nutr 2021;10(5):661671. doi: 10.21037/hbsn-21-260 trial. Hepatology 2014;60:1697-707.

54. Kudo M, Cheng AL, Park JW, et al. Orantinib versus placebo combined with transcatheter arterial chemoembolisation in patients with unresectable hepatocellular carcinoma (ORIENTAL): a randomised, double-blind, placebo-controlled, multicentre, phase 3 study. Lancet Gastroenterol Hepatol 2018;3:37-46.

55. Kudo M, Ueshima K, Ikeda $M$, et al. Randomised, multicentre prospective trial of transarterial chemoembolisation (TACE) plus sorafenib as compared with TACE alone in patients with hepatocellular carcinoma: TACTICS trial. Gut 2020;69:1492-501.

56. El-Khoueiry AB, Sangro B, Yau T, et al. Nivolumab in patients with advanced hepatocellular carcinoma (CheckMate 040): an open-label, non-comparative, phase $1 / 2$ dose escalation and expansion trial. Lancet 2017;389:2492-502.

57. Zhu AX, Finn RS, Edeline J, et al. Pembrolizumab in patients with advanced hepatocellular carcinoma previously treated with sorafenib (KEYNOTE-224): a non-randomised, open-label phase 2 trial. Lancet Oncol 2018;19:940-52.

58. Finn RS, Qin S, Ikeda M, et al. IMbrave150: Updated overall survival (OS) data from a global, randomized, open-label phase III study of atezolizumab (atezo) + bevacizumab (bev) versus sorafenib (sor) in patients (pts) with unresectable hepatocellular carcinoma (HCC). J Clin Oncol 2021;39:abstr 267. 